\title{
A Note on the Inverse Problem for a Fractional Parabolic Equation
}

\author{
Abdullah Said Erdogan and Hulya Uygun \\ Department of Mathematics, Fatih University, 34500 Buyukcekmece, Istanbul, Turkey \\ Correspondence should be addressed to Abdullah Said Erdogan, aserdogan@gmail.com \\ Received 15 May 2012; Accepted 8 July 2012 \\ Academic Editor: Ravshan Ashurov \\ Copyright (C 2012 A. S. Erdogan and H. Uygun. This is an open access article distributed under \\ the Creative Commons Attribution License, which permits unrestricted use, distribution, and \\ reproduction in any medium, provided the original work is properly cited. \\ For a fractional inverse problem with an unknown time-dependent source term, stability estimates \\ are obtained by using operator theory approach. For the approximate solutions of the problem, \\ the stable difference schemes which have first and second orders of accuracy are presented. The \\ algorithm is tested in a one-dimensional fractional inverse problem.
}

\section{Introduction}

Inverse problems arise in many fields of science and engineering such as ion transport problems, chromatography, and heat determination problems with an unknown internal energy source. Different typed of inverse problems have been investigated, and the main results obtained in this field of research were given by many researchers (see [1-10]). More than three centuries the theory of fractional derivatives developed mainly as a pure theoretical field of mathematics. Fractional integrals and derivatives appear in the theory of control of dynamical systems, when the controlled system or/and the controller is described by a fractional differential equation (see [11]). Recently, many application areas such as bioengineering applications, image and signal processing are also related to fractional calculus. Methods of solutions of problems and theory of fractional calculus have been studied by many researchers [11-28]. Among them finite difference method is used for solving several fractional differential equations (see [20, 22, 23, 27] and the references therein).

\subsection{Statement of the Problem}

Many scientists and researchers are trying to enhance mathematical models of real-life cases for investigating and understanding the behavior of them. Therefore, some phenomena have 
been modeled and investigated as fractional inverse problems (see [29-33] and the references therein). In this paper, we consider the fractional parabolic inverse problem with the Dirichlet condition

$$
\begin{gathered}
\frac{\partial u(t, x)}{\partial t}-a \frac{\partial^{2} u(t, x)}{\partial x^{2}}-D_{t}^{1 / 2} u(t, x)+\sigma u(t, x)=p(t) q(x)+f(t, x), \quad 0<x<\pi, 0<t \leq T, \\
u(t, 0)=u(t, \pi)=0, \quad 0 \leq t \leq T, \\
u(0, x)=\varphi(x), \quad 0 \leq x \leq \pi \\
u\left(t, x^{*}\right)=\rho(t), \quad 0<x^{*}<\pi
\end{gathered}
$$

where $u(t, x)$ and $p(t)$ are unknown functions, $a(x) \geq a>0$, and $\sigma>0$ is a sufficiently large number. Here, $D_{t}^{1 / 2}=D_{0+}^{1 / 2}$ is the standard Riemann-Liouville's derivative of order $1 / 2$.

Theorems on the stability of problem (1.1) are analyzed by assuming that $q(x)$ is a sufficiently smooth function, $q(0)=q(\pi)=0$ and $q\left(x^{*}\right) \neq 0$.

\section{Main Results}

In this section, stability estimates for the solution of (1.1) are investigated. For the mathematical substantiation, we introduce the Banach space $\stackrel{\circ}{C}^{\alpha}[0, \pi], \alpha \in(0,1)$, of all continuous functions $\phi(x)$ defined on $[0, \pi]$ with $\phi(0)=\phi(\pi)=0$ satisfying a Hölder condition for which the following norm is finite

$$
\|\phi\|_{C^{\circ}[0, \pi]}=\|\phi\|_{C[0, \pi]}+\sup _{0<x<x+h<\pi} \frac{|\phi(x+h)-\phi(x)|}{h^{\alpha}}
$$

where $C[0, \pi]$ is the space of all continuous function $\phi(x)$ defined on $[0, \pi]$ with the norm

$$
\|\phi\|_{C[0, \pi]}=\max _{0 \leq x \leq \pi}|\phi(x)| .
$$

With the help of a positive operator $A$, we introduce the fractional spaces $E_{\alpha}, 0<\alpha<1$, consisting of all $v$ in a Banach space $E$ for which the following norm is finite:

$$
\|v\|_{E_{\alpha}}=\|v\|_{E}+\sup _{\lambda>0} \lambda^{1-\alpha}\|A \exp \{-\lambda A\} v\|_{E}
$$

Throughout the paper, positive constants will be indicated by $M_{i}(\alpha, \beta, \ldots)$. Here variables are used to focus on the fact that the constant depends only on $\alpha, \beta, \ldots$ and the subindex $i$ is used to indicate a different constant. 
Theorem 2.1. Let $\varphi \in \stackrel{\circ}{C}^{2 \alpha+2}[0, \pi], F \in C\left([0, T], \stackrel{\circ}{C}^{2 \alpha}[0, \pi]\right)$, and $\rho^{\prime} \in C[0, T]$. Then for the solution of problem (1.1), the following coercive stability estimates

$$
\begin{aligned}
& \left\|u_{t}\right\|_{C\left([0, T], \stackrel{\circ}{C}^{2 \alpha}[0, \pi]\right)}+\|u\|_{C\left([0, T], \stackrel{\circ}{C}^{2 \alpha+2}[0, \pi]\right)} \leq M\left(x^{*}, q\right)\left\|\rho^{\prime}\right\|_{C[0, T]}+M\left(a, \delta, \sigma, \alpha, x^{*}, q, T\right) \\
& \times\left(\|\varphi\|_{C}^{\circ 2 \alpha+2}[0, \pi]+\|F\|_{C\left([0, T], C^{\circ 2 \alpha}[0, \pi]\right)}+\|\rho\|_{C[0, T]}\right), \\
& \|p\|_{C[0, T]} \leq M\left(x^{*}, q\right)\left\|\rho^{\prime}\right\|_{C[0, T]}+M\left(a, \delta, \sigma, \alpha, x^{*}, q, T\right) \\
& \times\left[\|\varphi\|_{\stackrel{\circ}{2}^{2 \alpha+2}[0, \pi]}+\|F\|_{C\left([0, T], \stackrel{\circ}{C}^{2 \alpha}[0, \pi]\right)}+\|\rho\|_{C[0, T]}\right]
\end{aligned}
$$

hold.

Proof. Let us search for the solution of inverse problem (1.1) in the following form (see [8]):

$$
u(t, x)=\eta(t) q(x)+w(t, x)
$$

where

$$
\eta(t)=\int_{0}^{t} p(s) d s
$$

Using the overdetermined condition, we get

$$
\begin{aligned}
\eta(t) & =\frac{\rho(t)-w\left(t, x^{*}\right)}{q\left(x^{*}\right)}, \\
p(t) & =\frac{\rho^{\prime}(t)-w_{t}\left(t, x^{*}\right)}{q\left(x^{*}\right)} .
\end{aligned}
$$

Using identity (2.8) and the triangle inequality, it follows that

$$
\begin{aligned}
|p(t)| & =\left|\frac{\rho^{\prime}(t)-w_{t}\left(t, x^{*}\right)}{q\left(x^{*}\right)}\right| \leq M\left(x^{*}, q\right)\left(\left|\rho^{\prime}(t)\right|+\left|w_{t}\left(t, x^{*}\right)\right|\right) \\
& \leq M\left(x^{*}, q\right)\left(\max _{0 \leq t \leq T}\left|\rho^{\prime}(t)\right|+\max _{0 \leq t \leq T} \max _{0 \leq x \leq \pi}\left|w_{t}(t, x)\right|\right) \\
& \leq M\left(x^{*}, q\right)\left(\max _{0 \leq t \leq T}\left|\rho^{\prime}(t)\right|+\max _{0 \leq t \leq T}\left\|w_{t}(t, \cdot)\right\|_{C^{2 \alpha}[0, \pi]}\right)
\end{aligned}
$$


for any $t, t \in[0, T]$. Here, $w(t, x)$ is the solution of the following problem:

$$
\begin{gathered}
\frac{\partial w(t, x)}{\partial t}-a \frac{\partial^{2} w(t, x)}{\partial x^{2}}-a \frac{\rho(t)-w\left(t, x^{*}\right)}{q\left(x^{*}\right)} \frac{d^{2} q(x)}{d x^{2}}-D_{t}^{1 / 2} w(t, x) \\
-\frac{D_{t}^{1 / 2} \rho(t)-D_{t}^{1 / 2} w\left(t, x^{*}\right)}{q\left(x^{*}\right)} q(x)+\sigma \frac{\rho(t)-w\left(t, x^{*}\right)}{q\left(x^{*}\right)} q(x) \\
+\sigma w(t, x)=f(t, x), \quad 0<x<\pi, \quad 0<t \leq T, \\
w(t, 0)=w(t, \pi)=0, \quad 0 \leq t \leq T, \\
w(0, x)=\varphi(x), \quad 0 \leq x \leq \pi .
\end{gathered}
$$

For simplicity, we assign

$$
\begin{gathered}
F(t, x)=\frac{a \rho(t)}{q\left(x^{*}\right)} \frac{d^{2} q(x)}{d x^{2}}-\frac{\sigma \rho(t)}{q\left(x^{*}\right)} q(x)+\frac{D_{t}^{1 / 2} \rho(t)}{q\left(x^{*}\right)} q(x)+f(t, x), \\
G(t, x)=Q_{1}\left(q, \rho, x, x^{*}, t\right) w\left(t, x^{*}\right)+Q_{2}\left(q, x, x^{*}\right) D_{t}^{1 / 2} w\left(t, x^{*}\right)+D_{t}^{1 / 2} w(t, x),
\end{gathered}
$$

where

$$
\begin{gathered}
Q_{1}\left(q, \rho, x, x^{*}, t\right)=\frac{1}{q\left(x^{*}\right)}\left(-a \frac{d^{2} q(x)}{d x^{2}}+\sigma \rho(t)\right), \\
Q_{2}\left(q, x, x^{*}\right)=-\frac{q(x)}{q\left(x^{*}\right)} .
\end{gathered}
$$

Note that functions $F(t, x), Q_{1}\left(q, \rho, x, x^{*}, t\right)$ and $Q_{2}\left(q, x, x^{*}\right)$ only contain given functions. Then, we can rewrite problem (2.10) as

$$
\begin{gathered}
\frac{\partial w(t, x)}{\partial t}-a \frac{\partial^{2} w(t, x)}{\partial x^{2}}+\sigma w(t, x)=F(t, x)+G(t, x), \quad 0<x<\pi, 0<t \leq T, \\
w(t, 0)=w(t, \pi)=0, \quad 0 \leq t \leq T, \\
w(0, x)=\varphi(x), \quad 0 \leq x \leq \pi .
\end{gathered}
$$

So, the end of proof of Theorem 2.1 is based on estimate (2.9) and the following theorem.

Theorem 2.2. For the solution of problem (2.10), the following coercive stability estimate

$$
\begin{aligned}
\left\|w_{t}\right\|_{\mathrm{O}^{2 \alpha}[0, \pi]} \leq & M\left(a, \delta, \sigma, \alpha, x^{*}, q, T\right) \\
& \times\left(\|\varphi\|_{C}{ }_{C}^{2 \alpha+2}[0, \pi]\right. \\
& \left.+\|F\|_{C\left([0, T], C^{\circ 2 \alpha}[0, \pi]\right)}+\|\rho\|_{C[0, T]}\right)
\end{aligned}
$$

holds. 
Proof. In a Banach space $E=\stackrel{\circ}{C}[0, \pi]$, with the help of the positive operator $A$ defined by

$$
A u=-a(x) \frac{\partial^{2} u(t, x)}{\partial x^{2}}+\sigma u,
$$

with

$$
D(A)=\left\{u(x): u, u^{\prime}, u^{\prime \prime} \in C[0, \pi], u(0)=u(\pi)=0\right\},
$$

where $\sigma$ is a positive constant, problem (2.10) can be written in the abstract form as an initialvalue problem

$$
\begin{gathered}
w_{t}+A w=F(t)+G(t), \quad 0<t \leq T, \\
w(0)=\varphi .
\end{gathered}
$$

By the Cauchy formula, the solution can be written as

$$
w(t)=e^{-t A} \varphi-\int_{0}^{t} e^{-(t-s) A}(F(s)+G(s)) d s .
$$

Applying the formula

$$
D_{t}^{1 / 2} u(t)=\int_{0}^{t} \frac{u^{\prime}(\zeta) d \xi}{\sqrt{\pi}(t-\xi)^{1 / 2}}
$$

we get the following presentation of the solution of abstract problem (2.17):

$$
\begin{aligned}
D^{1 / 2} w(t)= & -\int_{0}^{t} \frac{A e^{-\xi A} \varphi}{\sqrt{\pi}(t-\xi)^{1 / 2}} d \xi-\int_{0}^{t} \frac{F(\xi)}{\sqrt{\pi}(t-\xi)^{1 / 2}} d \xi \\
& -\int_{0}^{t} \frac{G(\xi)}{\sqrt{\pi}(t-\xi)^{1 / 2}} d \xi+\int_{0}^{t} \int_{0}^{\xi} \frac{A e^{-(\xi-s) A} F(s)}{\sqrt{\pi}(t-\xi)^{1 / 2}} d s d \xi \\
& +\int_{0}^{t} \int_{0}^{\xi} \frac{A e^{-(\xi-s) A} G(s)}{\sqrt{\pi}(t-\xi)^{1 / 2}} d s d \xi .
\end{aligned}
$$


Changing the order of integration, we obtain that

$$
\begin{aligned}
D^{1 / 2} w(t)= & -\int_{0}^{t} \frac{A e^{-\xi A} \varphi}{\sqrt{\pi}(t-\xi)^{1 / 2}} d \xi-\int_{0}^{t} \frac{F(\xi)}{\sqrt{\pi}(t-\xi)^{1 / 2}} d \xi \\
& +\int_{0}^{t} \int_{s}^{t} \frac{A e^{-(\xi-s) A}}{\sqrt{\pi}(t-\xi)^{1 / 2}} d \xi F(s) d s-\int_{0}^{t} \frac{G(\xi)}{\sqrt{\pi}(t-\xi)^{1 / 2}} d \xi \\
& +\int_{0}^{t} \int_{s}^{t} \frac{A e^{-(\xi-s) A}}{\sqrt{\pi}(t-\xi)^{1 / 2}} d \xi G(s) d s=\sum_{k=1}^{5} J_{k},
\end{aligned}
$$

where

$$
\begin{gathered}
J_{1}(t)=-\int_{0}^{t} \frac{A e^{-\xi A} \varphi}{\sqrt{\pi}(t-p)^{1 / 2}} d \xi, \\
J_{2}(t)=-\int_{0}^{t} \frac{F(\xi)}{\sqrt{\pi}(t-\xi)^{1 / 2}} d \xi, \\
J_{3}(t)=\int_{0}^{t} \int_{s}^{t} \frac{A e^{-(\xi-s) A}}{\sqrt{\pi}(t-\xi)^{1 / 2}} d \xi F(s) d s, \\
J_{4}(t)=-\int_{0}^{t} \frac{G(\xi)}{\sqrt{\pi}(t-\xi)^{1 / 2}} d \xi, \\
J_{5}(t)=\int_{0}^{t} \int_{s}^{t} \frac{A e^{-(\xi-s) A}}{\sqrt{\pi}(t-\xi)^{1 / 2}} d \xi G(s) d s .
\end{gathered}
$$

Now, we estimate $J_{k}(t), k=1,2,3,4,5$ separately. It is known that [13]

$$
\left\|A^{\alpha} e^{-t A}\right\|_{E \rightarrow E} \leq M, \quad 0 \leq \alpha \leq 1
$$

Since operators $A$ and $\exp (-t A)$ commute,

$$
\left\|A e^{-t A} \varphi\right\|_{E_{\alpha}} \leq\left\|e^{-t A}\right\|_{E_{\alpha} \rightarrow E_{\alpha}}\|A \varphi\|_{E_{\alpha}} \leq\left\|e^{-t A}\right\|_{E \rightarrow E}\|A \varphi\|_{E_{\alpha}} .
$$

Applying the definition of norm of the spaces $E_{\alpha}$ and (2.23) and (2.24), we get

$$
\left\|J_{1}(t)\right\|_{E_{\alpha}}=\left\|\int_{0}^{t} \frac{A e^{-\xi A} \varphi}{\sqrt{\pi}(t-p)^{1 / 2}} d \xi\right\|_{E_{\alpha}} \leq M_{1}\|A \varphi\|_{E_{\alpha}}
$$


for any $t, t \in[0, T]$. Estimation of $J_{2}(t)$ is as follows:

$$
\begin{aligned}
\left\|J_{2}(t)\right\|_{E_{\alpha}} & =\left\|\int_{0}^{t} \frac{F(\xi)}{\sqrt{\pi}(t-\xi)^{1 / 2}} d \xi\right\|_{E_{\alpha}} \\
& \leq\|F(t)\|_{C\left(E_{\alpha}\right)} \int_{0}^{t} \frac{1}{\sqrt{\pi}(t-\xi)^{1 / 2}} d \xi \leq M_{2}\|F\|_{C\left(E_{\alpha}\right)} .
\end{aligned}
$$

Let us estimate $J_{3}(t)$ :

$$
\left\|J_{3}(t)\right\|_{E_{\alpha}}=\left\|\int_{0}^{t} \int_{s}^{t} \frac{A e^{-(\xi-s) A}}{\sqrt{\pi}(t-\xi)^{1 / 2}} d \xi F(s) d s\right\|_{E_{\alpha}} \leq\left\|\int_{0}^{t} \int_{s}^{t} \frac{A e^{-(\xi-s) A}}{\sqrt{\pi}(t-\xi)^{1 / 2}} d \xi d s\right\|_{E_{\alpha} \rightarrow E_{\alpha}}\|F\|_{C\left(E_{\alpha}\right)}
$$

It is proven that (see [28])

$$
\left\|\int_{s}^{t} \frac{A e^{-(\xi-s) A}}{\sqrt{\pi}(t-\xi)^{1 / 2}} d \xi\right\|_{E \rightarrow E} \leq \frac{M}{\sqrt{t-s}}
$$

Using the definition of norm of the spaces $E_{\alpha}$, we can obtain that

$$
\begin{aligned}
\left\|\int_{0}^{t} \int_{s}^{t} \frac{A e^{-(\xi-s) A}}{\sqrt{\pi}(t-\xi)^{1 / 2}} d \xi d s\right\|_{E_{\alpha} \rightarrow E_{\alpha}} & \int_{0}^{t}\left\|\int_{s}^{t} \frac{A e^{-(t-s) A}}{\sqrt{\pi}(t-\xi)^{1 / 2}} d \xi\right\|_{E \rightarrow E} d s \\
& +\sup _{\lambda>0} \int_{0}^{t}\left\|\int_{s}^{t} \lambda^{1-\alpha} A e^{-\lambda A} \frac{A e^{-(t-s) A}}{\sqrt{\pi}(t-\xi)^{1 / 2}} d \xi\right\|_{E \rightarrow E} d s .
\end{aligned}
$$

Using estimates (2.23) and (2.28), we get

$$
\begin{aligned}
\left\|J_{3}(t)\right\|_{E_{\alpha}} & \leq\left\|\int_{0}^{t} \int_{s}^{t} \frac{A e^{-(\xi-s) A}}{\sqrt{\pi}(t-\xi)^{1 / 2}} d \xi d s\right\|_{E_{\alpha} \rightarrow E_{\alpha}}\|F\|_{C\left(E_{\alpha}\right)} \\
& \leq M_{3}\|F\|_{C\left(E_{\alpha}\right)} .
\end{aligned}
$$

Expanding $G(s)$, estimation of $J_{4}(t)$ is as follows:

$$
\begin{aligned}
\left\|J_{4}(t)\right\|_{E_{\alpha}} \leq & \int_{0}^{t}\left\|\frac{Q_{1}\left(q, \rho, x, x^{*}, t\right) w\left(\xi, x^{*}\right)}{\sqrt{\pi}(t-\xi)^{1 / 2}}\right\|_{E_{\alpha}} d \xi \\
& +\int_{0}^{t}\left\|\frac{Q_{2}\left(q, x, x^{*}\right) D_{t}^{1 / 2} w\left(\xi, x^{*}\right)}{\sqrt{\pi}(t-\xi)^{1 / 2}}\right\|_{E_{\alpha}} d \xi+\int_{0}^{t}\left\|\frac{D_{t}^{1 / 2} w(\xi, x)}{\sqrt{\pi}(t-\xi)^{1 / 2}}\right\|_{E_{\alpha}} d \xi .
\end{aligned}
$$


It is known that (see [34])

$$
\|w\|_{E_{\alpha}} \leq M\left\|D_{t}^{1 / 2} w\right\|_{E_{\alpha}}
$$

Since $Q_{1}\left(q, \rho, x, x^{*}, t\right)$ and $Q_{2}\left(q, x, x^{*}\right)$ are known functions, it is easy to see that

$$
\left\|J_{4}(t)\right\|_{E_{\alpha}} \leq M_{4}\left(q, \rho, x, x^{*}, T\right) \int_{0}^{t} \frac{1}{\sqrt{\pi}(t-\xi)^{1 / 2}}\left\|D_{t}^{1 / 2} w(\xi)\right\|_{E_{\alpha}} d \xi
$$

Estimation of $J_{5}(t)$ can be given similar to the estimation of $J_{4}(t)$. By (2.23) and (2.32),

$$
\begin{aligned}
\left\|J_{5}(t)\right\|_{E_{\alpha}} & \leq \int_{0}^{t} \int_{s}^{t} \frac{A e^{-(\xi-s) A}}{\sqrt{\pi}(t-\xi)^{1 / 2}} d \xi G(s) d s \\
& \leq M_{5}\left(q, \rho, x, x^{*}, T\right) \int_{0}^{t}\left\|D_{t}^{1 / 2} w(s)\right\|_{E_{\alpha}} d s .
\end{aligned}
$$

Finally combining estimates (2.25), (2.26), (2.30), (2.33), and (2.34), we get

$$
\begin{aligned}
\left\|D_{t}^{1 / 2} w\right\|_{E_{\alpha}} \leq & M_{1}\|A \varphi\|_{E_{\alpha}}+\left(M_{2}+M_{3}\right)\|F\|_{C\left(E_{\alpha}\right)} \\
& +\int_{0}^{t}\left(\frac{M_{4}}{\sqrt{\pi}(t-\xi)^{1 / 2}}+M_{5}\right)\left\|D_{t}^{1 / 2} w(s)\right\|_{E_{\alpha}} d s .
\end{aligned}
$$

Using the Gronwall's inequality, we can write

$$
\left\|D_{t}^{1 / 2} w\right\|_{E_{\alpha}} \leq e^{M_{6}}\left(M_{1}\|A \varphi\|_{E_{\alpha}}+M_{7}\|F\|_{C\left(E_{\alpha}\right)}\right) .
$$

From the last estimate, we can obtain the estimate for $w_{t}(t)$ by using problem (2.17) and wellposedness of the Cauchy problem in $C\left(E_{\alpha}\right)$ (see [35]). So the following theorem finishes the proof of Theorem 2.2.

Theorem 2.3 (see, [36]). For $0<\alpha<1 / 2$, the norms of the spaces $E_{\alpha}(C[0, \pi], A)$ and $C^{2 \alpha}[0, \pi]$ are equivalent. 


\section{Numerical Results}

We have not been able to obtain a sharp estimate for the constants figuring in the stability inequalities. So we will provide the following results of numerical experiments of the following problem:

$$
\begin{gathered}
\frac{\partial u(t, x)}{\partial t}=\frac{\partial^{2} u(t, x)}{\partial x^{2}}-u(t, x)+D_{t}^{1 / 2} u(t, x)+p(t) \sin x+f(t, x) \\
f(t, x)=\left(-3 t-\frac{1}{\sqrt{\pi}} t^{-1 / 2}+\frac{2}{\sqrt{\pi}} t^{1 / 2}\right) \sin x, \quad x \in(0, \pi), t \in(0,1] \\
u(0, x)=\sin x, \quad x \in[0, \pi] \\
u(t, 0)=u(t, \pi)=0, \quad t \in[0,1] \\
u\left(t, \frac{\pi}{2}\right)=1-t .
\end{gathered}
$$

The exact solution of the given problem is $u(t, x)=(1-t) \sin x$ and for the control parameter $p(t)$ is $1+t$.

\subsection{The First Order of Accuracy Difference Scheme}

For the approximate solution of the problem (3.1), the Rothe difference scheme

$$
\begin{gathered}
\frac{u_{n}^{k}-u_{n}^{k-1}}{\tau}=\frac{u_{n+1}^{k}-2 u_{n}^{k}+u_{n-1}^{k}}{h^{2}}-u_{n}^{k}+D_{\tau}^{1 / 2} u_{n}^{k}+p^{k} q_{n}+f\left(t_{k}, x_{n}\right), \\
f\left(t_{k}, x_{n}\right)=\left(-3 t_{k}-\frac{1}{\sqrt{\pi}} t_{k}^{-1 / 2}+\frac{2}{\sqrt{\pi}} t_{k}^{1 / 2}\right) \sin x_{n}, \\
p^{k}=p\left(t_{k}\right), \quad q_{n}=\sin x_{n}, \quad x_{n}=n h, \quad t_{k}=k \tau, \\
1 \leq k \leq N, \quad 1 \leq n \leq M-1, \quad M h=\pi, \quad N \tau=1, \\
u_{n}^{0}=\sin x_{n}, \quad 0 \leq n \leq M, \\
u_{0}^{k}=u_{M}^{k}=0, \quad 0 \leq k \leq N, \\
u_{s}^{k}=\rho\left(t_{k}\right), \quad \rho\left(t_{k}\right)=1-t_{k}, \quad 0 \leq k \leq N, \quad s=\left\lfloor\frac{\pi}{2 h}\right\rfloor
\end{gathered}
$$


where $\lfloor x\rfloor$ denotes greatest integer less than $x$ is constructed. Throughout the paper, let us denote

$$
\begin{gathered}
\rho\left(t_{k}\right)=1-t_{k}, \quad q_{n}=\sin x_{n}, \\
t_{k}=\left\{t_{k}=k \tau, 0 \leq k \leq N, N \tau=1\right\}, \\
x_{n}=\left\{x_{n}=n h, 0 \leq n \leq M-1, M h=\pi\right\}, \\
f\left(t_{k}, x_{n}\right)=\left(-3 t_{k}-\frac{1}{\sqrt{\pi}} t_{k}^{-1 / 2}+\frac{2}{\sqrt{\pi}} t_{k}^{1 / 2}\right) \sin x_{n}, \\
F\left(t_{k}, x_{n}\right)=\frac{\rho\left(t_{k}\right)}{\sin \left(x_{s}\right)}\left(\frac{\sin \left(x_{n+1}\right)-2 \sin \left(x_{n}\right)+\sin \left(x_{n-1}\right)}{h^{2}}-\sin \left(x_{n}\right)\right) \\
-\frac{1}{\sqrt{\pi}} \sum_{m=1}^{k} \frac{\Gamma(k-m+(1 / 2))}{(k-m) !} \frac{\tau^{1 / 2}}{\sin \left(x_{s}\right)} \sin \left(x_{n}\right)+f\left(t_{k}, x_{n}\right) .
\end{gathered}
$$

We search the solution of (3.2) in the following form:

$$
u_{n}^{k}=\eta^{k} q_{n}+w_{n}^{k}
$$

where

$$
\eta^{k}=\sum_{i=1}^{k} p^{i} \tau, \quad 1 \leq k \leq N, \eta^{0}=0
$$

Moreover for the interior grid point $u_{s}^{k}$, we have that

$$
u_{s}^{k}=\eta^{k} q_{s}+w_{s}^{k}=\rho\left(t_{k}\right)
$$

From (3.4), (3.5), and the condition $u_{s}^{k}=\rho\left(t_{k}\right)$, it follows that

$$
\begin{gathered}
\eta^{k}=\frac{\rho\left(t_{k}\right)-w_{s}^{k}}{q_{s}}, \\
p^{k}=\frac{\eta^{k}-\eta^{k-1}}{\tau}, \quad 1 \leq k \leq N, \\
u_{n}^{k}=\frac{\rho\left(t_{k}\right)-w_{s}^{k}}{q_{s}} q_{n}+w_{n}^{k}, \quad 0 \leq k \leq N, 0 \leq n \leq M,
\end{gathered}
$$


where $w_{n}^{k}, 0 \leq k \leq N, 0 \leq n \leq M$ is the solution of the difference scheme

$$
\begin{aligned}
\frac{w_{n}^{k}-w_{n}^{k-1}}{\tau}= & \frac{w_{n+1}^{k}-2 w_{n}^{k}+w_{n-1}^{k}}{h^{2}}-w_{n}^{k} \\
& -\frac{w_{s}^{k}}{\sin \left(x_{s}\right)}\left(\frac{\sin \left(x_{n+1}\right)-2 \sin \left(x_{n}\right)+\sin \left(x_{n-1}\right)}{h^{2}}-\sin \left(x_{n}\right)\right) \\
& \quad-\frac{1}{\sqrt{\pi}} \sum_{m=1}^{k} \frac{\Gamma(k-m+(1 / 2))}{(k-m) !}\left(\frac{w_{s}^{m}-w_{s}^{m-1}}{\sin \left(x_{s}\right) \tau^{1 / 2}} \sin \left(x_{n}\right)-\frac{w_{n}^{m}-w_{n}^{m-1}}{\tau^{1 / 2}}\right) \\
& +F\left(t_{k}, x_{n}\right), \quad 1 \leq k \leq N, 1 \leq n \leq M-1, \\
w_{0}^{k}= & w_{M}^{k}=0, \quad 0 \leq k \leq N, \\
w_{n}^{0}= & \sin \left(x_{n}\right), \quad 0 \leq n \leq M .
\end{aligned}
$$

First, applying the first order of accuracy difference scheme (3.10), we obtain $(M+1) \times(M+1)$ system of linear equations and we write them in the matrix form

$$
A w^{k}+\sum_{j=0}^{k-1} B_{j} w^{j}=D \varphi^{k}, \quad 1 \leq k \leq N, w^{0}=\left\{\sin \left(x_{n}\right)\right\}_{n=0}^{M}
$$

where

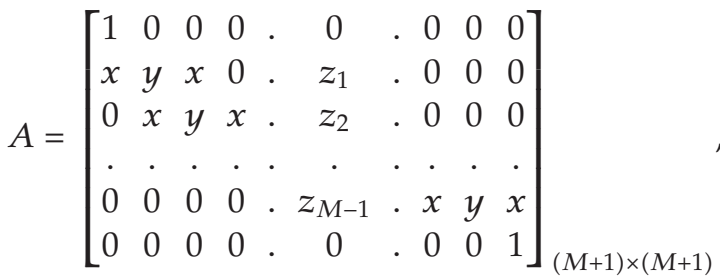

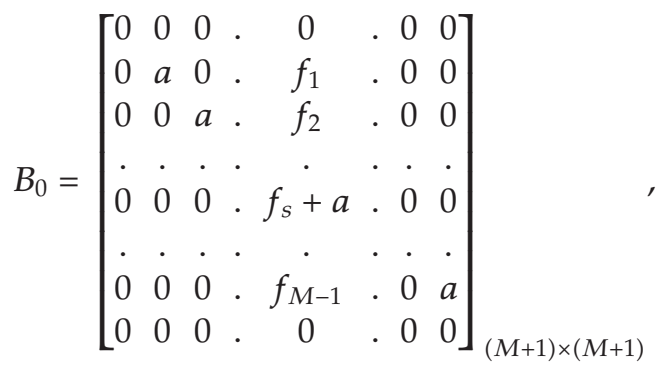




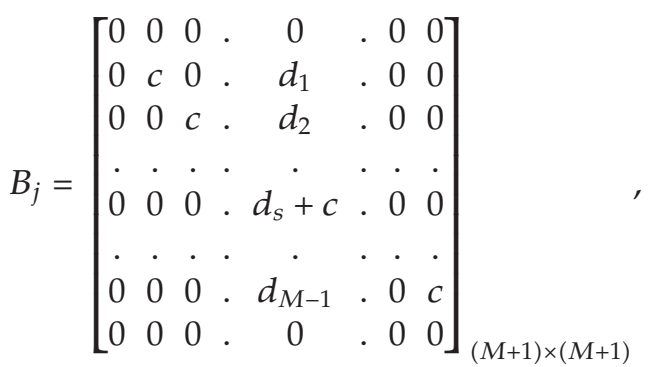

for any $j=1,2, \ldots, k-2$, and

$$
B_{k-1}=\left[\begin{array}{cccccccc}
0 & 0 & 0 & . & 0 & . & 0 & 0 \\
0 & v & 0 & . & c_{1} & . & 0 & 0 \\
0 & 0 & v & . & c_{2} & . & 0 & 0 \\
. & . & . & . & . & . & . & . \\
0 & 0 & 0 & . & c_{s}+v & . & 0 & 0 \\
. & . & . & . & . & . & . & . \\
0 & 0 & 0 & . & c_{M-1} & . & 0 & v \\
0 & 0 & 0 & . & 0 & . & 0 & 0
\end{array}\right]_{(M+1) \times(M+1)}
$$

Here, for any $n=1,2, \ldots, M-1$,

$$
\begin{aligned}
x & =-\frac{1}{h^{2}}, \quad y=\frac{1}{\tau}+\frac{2}{h^{2}}+1+\frac{1}{\sqrt{\tau}}, \\
z_{n} & =\frac{\sin \left(x_{n+1}\right)-2 \sin \left(x_{n}\right)+\sin \left(x_{n-1}\right)}{\sin \left(x_{s}\right) h^{2}}-\frac{\sin \left(x_{n}\right)}{\sin \left(x_{s}\right)}-\frac{\sin \left(x_{n}\right)}{\sin \left(x_{s}\right) \sqrt{\tau}}, \quad \text { in }(s+1) \text { th column, } \\
a & =\frac{\Gamma(k-1 / 2)}{\sqrt{\tau \pi}(k-1) !}, \quad f_{n}=-\frac{\sin \left(x_{n}\right) \Gamma(k-1 / 2)}{\sqrt{\tau \pi} \sin \left(x_{s}\right)(k-1) !}, \\
c & =\frac{1}{\sqrt{\tau \pi}}\left(\frac{\Gamma(k-j-1 / 2)}{(k-j-1) !}-\frac{\Gamma(k-j+1 / 2)}{(k-j) !}\right), \\
d_{n} & =\frac{\sin \left(x_{n}\right)}{\sqrt{\tau \pi} \sin \left(x_{s}\right)}\left(\frac{\Gamma(k-j-1 / 2)}{(k-j-1) !}-\frac{\Gamma(k-j+1 / 2)}{(k-j) !}\right), \\
v & =-\frac{1}{\sqrt{\tau}}-\frac{1}{\tau}, \quad c_{n}=\frac{\sin \left(x_{n}\right)}{\sqrt{\tau} \sin \left(x_{s}\right)},
\end{aligned}
$$




$$
\begin{aligned}
w^{r}= & {\left[\begin{array}{c}
w_{0}^{r} \\
\vdots \\
w_{M}^{r}
\end{array}\right]_{(M+1) \times 1} \quad \text { for any } r=0,1, \ldots, k, } \\
\varphi^{k}= & {\left[\begin{array}{c}
0 \\
\phi_{1}^{k} \\
\vdots \\
\phi_{M-1}^{k} \\
0
\end{array}\right]_{(M+1) \times 1}, } \\
\phi_{n}^{k}= & \left(\frac{\rho\left(t_{k}\right)}{\sin \left(x_{s}\right)} \frac{\sin \left(x_{n+1}\right)-2 \sin \left(x_{n}\right)+\sin \left(x_{n-1}\right)}{h^{2}}-\frac{\rho\left(t_{k}\right)}{\sin \left(x_{s}\right)} \sin \left(x_{n}\right)\right) \\
& -\frac{1}{\sqrt{\pi}} \sum_{m=1}^{k} \frac{\Gamma(k-m+1 / 2)}{(k-m) !} \frac{\tau^{1 / 2}}{\sin \left(x_{s}\right)} \sin \left(x_{n}\right)+f\left(t_{k}, x_{n}\right),
\end{aligned}
$$

and $D$ is $(M+1) \times(M+1)$ identity matrix. Using (3.11), we can obtain that

$$
w^{k}=A^{-1}\left(D \varphi^{k}-\sum_{j=0}^{k-1} B_{j} w^{j}\right), \quad k=1,2, \ldots, N, \quad w^{0}=\left\{\sin x_{n}\right\}_{n=0}^{M} .
$$

To solve the resulting difference equations, we apply the method given in (3.15) step by step for $k=1,2, \ldots, N$. For the evaluation of $w^{r}, r=2,3, \ldots, N, w^{r-1}$ is needed. It is obtained in the previous step. Then, the solution pairs $(u, p)$ are obtained by using the last formulas (3.9) and (3.8).

\subsection{The Second Order of Accuracy Difference Scheme}

For the approximate solution of the problem (3.1), the Crank-Nicholson difference scheme

$$
\begin{gathered}
\frac{u_{n}^{k}-u_{n}^{k-1}}{\tau}=\frac{u_{n+1}^{k}-2 u_{n}^{k}+u_{n-1}^{k}}{2 h^{2}}+\frac{u_{n+1}^{k-1}-2 u_{n}^{k-1}+u_{n-1}^{k-1}}{2 h^{2}} \\
-\frac{u_{n}^{k}+u_{n}^{k-1}}{2}+\frac{p^{k}+p^{k-1}}{2} q_{n}+D_{\tau}^{1 / 2} u\left(t_{k}-\frac{\tau}{2}, x_{n}\right)+f\left(t_{k}-\frac{\tau}{2}, x_{n}\right), \\
p^{k}=p\left(t_{k}\right), \quad 1 \leq k \leq N, 1 \leq n \leq M-1 \\
u_{n}^{0}=\sin \left(x_{n}\right), \quad 0 \leq n \leq M, \\
u_{0}^{k}=u_{M}^{k}=0, \quad 0 \leq k \leq N, \\
u_{s}^{k}+\frac{u_{s+1}^{k}-u_{s}^{k}}{h}\left(x^{*}-s h\right)=\rho\left(t_{k}\right), \quad 0 \leq k \leq N, s=\left\lfloor\frac{\pi}{2 h}\right\rfloor
\end{gathered}
$$

is constructed. 
Here,

$$
\Gamma\left(k-r+\frac{1}{2}\right)=\int_{0}^{\infty} t^{k-r+1 / 2} e^{-t} d t
$$

Moreover, applying the second order of approximation formula for

$$
D_{t}^{1 / 2} u\left(t_{k}-\frac{\tau}{2}\right)=\frac{1}{\Gamma(1 / 2)} \int_{0}^{t_{k}-\tau / 2}\left(t_{k}-\frac{\tau}{2}-s\right)^{-1 / 2} u^{\prime}(s) d s
$$

it is obtained (see [27])

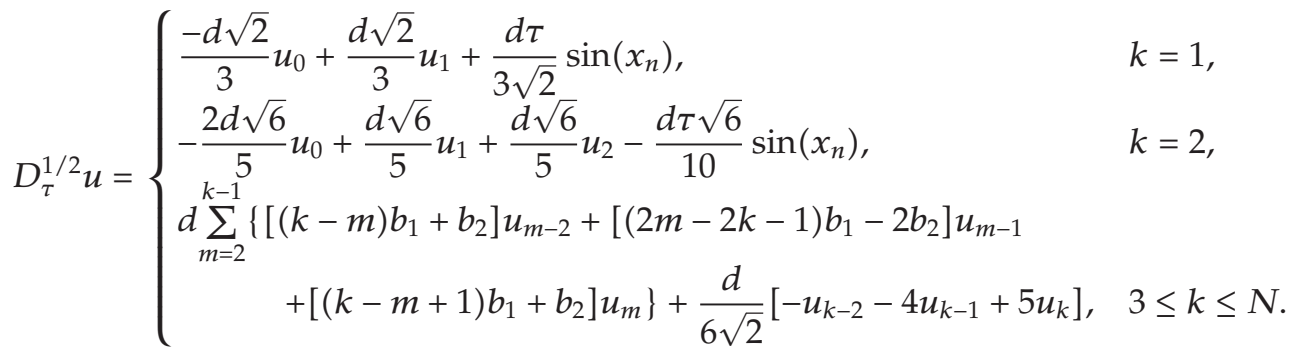

Here and throughout the paper,

$$
\begin{gathered}
D_{t}^{1 / 2} u=D_{\tau}^{1 / 2} u\left(t_{k}-\frac{\tau}{2}, x_{n}\right), \\
d=\frac{2}{\sqrt{\pi \tau}}, \quad b_{1}=\sqrt{k-m+\frac{1}{2}}-\sqrt{k-m-\frac{1}{2}} \\
b_{2}=-\frac{1}{3}\left(\left(k-m+\frac{1}{2}\right)^{3 / 2}-\left(k-m-\frac{1}{2}\right)^{3 / 2}\right) .
\end{gathered}
$$

We search the solution of (3.16) in the following form:

$$
u_{n}^{k}=\eta^{k} q_{n}+w_{n}^{k}
$$

where

$$
\eta^{k}=\sum_{i=1}^{k} \frac{p^{i}+p^{i-1}}{2} \tau, \quad 1 \leq k \leq N, \eta^{0}=0
$$


We have that

$$
\begin{aligned}
u_{s}^{k}+\frac{u_{s+1}^{k}-u_{s}^{k}}{h}\left(x^{*}-s h\right)= & \eta^{k}\left(\left(1-\frac{x^{*}-s h}{h}\right) q_{s}+\frac{x^{*}-s h}{h} q_{s+1}\right) \\
& +\left(1-\frac{x^{*}-s h}{h}\right) w_{s}^{k}+\frac{x^{*}-s h}{h} w_{s+1}^{k}=\rho\left(t_{k}\right) .
\end{aligned}
$$

Let us denote

$$
y=\frac{x^{*}-s h}{h}=\frac{x^{*}}{h}-\left\lfloor\frac{x^{*}}{h}\right\rfloor h,
$$

where $0 \leq y<1$. Then, one can write

$$
\eta^{k}=\frac{\rho\left(t_{k}\right)-(1-y) w_{s}^{k}-y w_{s+1}^{k}}{(1-y) q_{s}+y q_{s+1}}
$$

So the values of $\left(p\left(t_{k}\right)+p\left(t_{k-1}\right)\right) / 2,1 \leq k \leq N$ can be obtained by the following formula:

$$
\frac{p^{k}+p^{k-1}}{2}=\frac{\left(\rho\left(t_{k}\right)-\rho\left(t_{k-1}\right)\right) / \tau-(1-y)\left(\left(w_{s}^{k}-w_{s}^{k-1}\right) / \tau\right)-y\left(\left(w_{s+1}^{k}-w_{s+1}^{k-1}\right) / \tau\right)}{(1-y) q_{s}+y q_{s+1}} .
$$

Let $w^{r}$ denote

$$
w^{r}=\left[\begin{array}{c}
w_{0}^{r} \\
\vdots \\
w_{M}^{r}
\end{array}\right]_{(M+1) \times 1} \quad \text { for } r=0,1, \ldots, N
$$

For $k=1$, one can show that $w^{1}$ is the solution of the difference scheme

$$
\begin{aligned}
\frac{w_{n}^{1}-w_{n}^{0}}{\tau}= & \frac{w_{n+1}^{1}-2 w_{n}^{1}+w_{n-1}^{1}}{2 h^{2}}+\frac{w_{n+1}^{0}-2 w_{n}^{0}+w_{n-1}^{0}}{2 h^{2}} \\
& -\frac{w_{n}^{1}+w_{n}^{0}}{2}+\left(\frac{q_{n+1}-2 q_{n}+q_{n-1}}{2 h^{2}}-\frac{q_{n}}{2}\right)
\end{aligned}
$$




$$
\begin{aligned}
& \quad \times\left(\frac{\rho\left(t_{1}\right)-(1-y) w_{s}^{1}-y w_{s+1}^{1}}{(1-y) q_{s}+y q_{s+1}}+\frac{\rho\left(t_{0}\right)-(1-y) w_{s}^{0}-y w_{s+1}^{0}}{(1-y) q_{s}+y q_{s+1}}\right) \\
& +\frac{d \sqrt{2}}{3}\left(\frac{\rho\left(t_{1}\right)-(1-y) w_{s}^{1}-y w_{s+1}^{1}}{(1-y) q_{s}+y q_{s+1}} q(n)+w_{n}^{1}\right) \\
& \quad-\frac{d \sqrt{2}}{3}\left(\frac{\rho\left(t_{0}\right)-(1-y) w_{s}^{0}-y w_{s+1}^{0}}{(1-y) q_{s}+y q_{s+1}} q(n)+w_{n}^{0}\right) \\
& +\frac{d \tau}{3 \sqrt{2}} q(n)+f\left(t_{1}-\frac{\tau}{2}, x_{n}\right), \quad 1 \leq n \leq M-1, \\
& w_{0}^{1}=w_{M}^{1}=0, \quad \\
& w_{n}^{0}=\sin \left(x_{n}\right), \quad 0 \leq n \leq M .
\end{aligned}
$$

We have the system of linear equations and we write them in the matrix form

$$
A_{1} w^{1}+B_{1} w^{0}=D \varphi^{1},
$$

where

$$
\begin{aligned}
& A_{1}=\left[\begin{array}{ccccccccccc}
1 & 0 & 0 & 0 & \cdot & 0 & \cdot & \cdot & 0 & 0 & 0 \\
a & y_{1} & a & 0 & \cdot & l_{1} & c_{1} & \cdot & 0 & 0 & 0 \\
0 & a & y_{1} & a & \cdot & l_{2} & c_{2} & \cdot & 0 & 0 & 0 \\
\cdot & \cdot & \cdot & \cdot & \cdot & \cdot & \cdot & \cdot & \cdot & \cdot & \cdot \\
0 & 0 & 0 & a & \cdot & l_{s}+a & c_{S} & \cdot & 0 & 0 & 0 \\
0 & 0 & 0 & 0 & \cdot & l_{s+1}+y_{1} & c_{S+1}+a & \cdot & 0 & 0 & 0 \\
\cdot & \cdot & \cdot & \cdot & \cdot & \cdot & \cdot & \cdot & \cdot & \cdot & \cdot \\
0 & 0 & 0 & 0 & \cdot & l_{M-1} & c_{M-1} & \cdot & a & y_{1} & a \\
0 & 0 & 0 & 0 & \cdot & 0 & 0 & \cdot & 0 & 0 & 1
\end{array}\right]_{(M+1) \times(M+1)}
\end{aligned}
$$

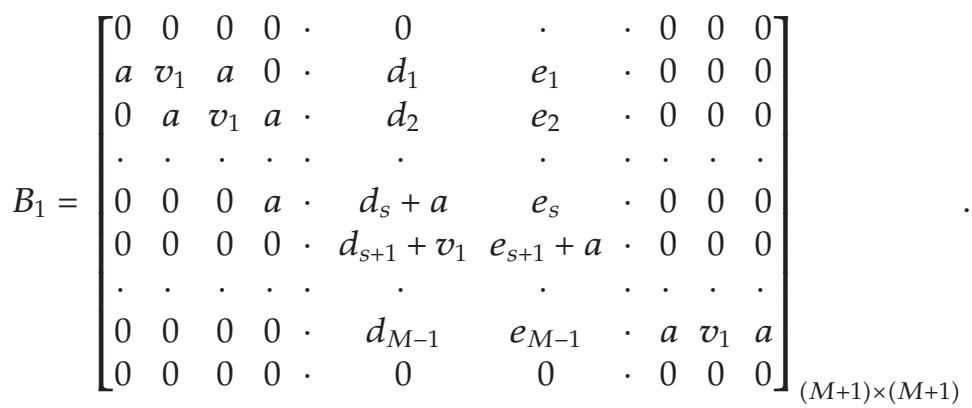


Here, for any $n=1,2, \ldots, M-1$,

$$
\begin{aligned}
& a=\left(-\frac{1}{2 h^{2}}\right), \quad y_{1}=\left(\frac{1}{\tau}+\frac{1}{h^{2}}+\frac{1}{2}-d \frac{\sqrt{2}}{3}\right), \\
& v_{1}=\left(-\frac{1}{\tau}+\frac{1}{h^{2}}+\frac{1}{2}+d \frac{\sqrt{2}}{3}\right) \\
& l_{n}=\frac{\left(q_{n+1}-2 q_{n}+q_{n-1}\right)(1-y)}{2 h^{2}\left((1-y) q_{s}+y q_{s+1}\right)}+\frac{q_{n}(1-y)}{2\left((1-y) q_{s}+y q_{s+1}\right)}+d \frac{\sqrt{2}}{3} q_{n}, \\
& c_{n}=\frac{\left(q_{n+1}-2 q_{n}+q_{n-1}\right) y}{2 h^{2}\left((1-y) q_{s}+y q_{s+1}\right)}+\frac{q_{n} y}{2\left((1-y) q_{s}+y q_{s+1}\right)}+d \frac{\sqrt{2}}{3} q_{n}, \\
& d_{n}=\frac{\left(q_{n+1}-2 q_{n}+q_{n-1}\right)(1-y)}{2 h^{2}\left((1-y) q_{s}+y q_{s+1}\right)}+\frac{q_{n}(1-y)}{2\left((1-y) q_{s}+y q_{s+1}\right)}-d \frac{\sqrt{2}}{3} q_{n}, \\
& e_{n}=\frac{\left(q_{n+1}-2 q_{n}+q_{n-1}\right) y}{2 h^{2}\left((1-y) q_{s}+y q_{s+1}\right)}+\frac{q_{n} y}{2\left((1-y) q_{s}+y q_{s+1}\right)}-d \frac{\sqrt{2}}{3} q_{n} \\
& \varphi^{1}=\left[\begin{array}{c}
0 \\
\phi_{1}^{1} \\
\vdots \\
\phi_{M-1}^{1} \\
0
\end{array}\right]_{(M+1) \times 1}, \\
& \phi_{n}^{1}=\left(\frac{\sin \left(x_{n+1}\right)-2 \sin \left(x_{n}\right)+\sin \left(x_{n-1}\right)}{2 h^{2}}-\frac{\sin \left(x_{n}\right)}{2}\right) \frac{\rho\left(t_{1}\right)+\rho\left(t_{0}\right)}{(1-y) q_{s}+y q_{s+1}} \\
& +\frac{d \sqrt{2} q_{n}}{3} \frac{\rho\left(t_{1}\right)-\rho\left(t_{0}\right)}{(1-y) q_{s}+y q_{s+1}}+\frac{d \tau}{3 \sqrt{2}} q_{n}+f\left(t_{1}-\frac{\tau}{2}, x_{n}\right)
\end{aligned}
$$

and $D$ is $(M+1) \times(M+1)$ identity matrix. Using (3.29), we can obtain that

$$
w^{1}=A_{1}^{-1}\left(D \varphi^{1}-B_{1} w^{0}\right), \quad w^{0}=\left\{\sin x_{n}\right\}_{n=0}^{M} .
$$

For $k=2, w^{2}$ is the solution of the difference scheme

$$
\begin{aligned}
& \frac{w_{n}^{2}-w_{n}^{1}}{\tau}=\frac{w_{n+1}^{2}-2 w_{n}^{2}+w_{n-1}^{2}}{2 h^{2}}-\frac{w_{n}^{2}+w_{n}^{1}}{2}+ \frac{w_{n+1}^{1}-2 w_{n}^{1}+w_{n-1}^{1}}{2 h^{2}} \\
&+\left(\frac{q_{n+1}-2 q_{n}+q_{n-1}}{2 h^{2}}-\frac{q_{n}}{2}\right)\left(\frac{\rho\left(t_{2}\right)-(1-y) w_{s}^{2}-y w_{s+1}^{2}}{(1-y) q_{s}+y q_{s+1}}\right. \\
&\left.+\frac{\rho\left(t_{1}\right)-(1-y) w_{s}^{1}-y w_{s+1}^{1}}{(1-y) q_{s}+y q_{s+1}}\right)
\end{aligned}
$$




$$
\begin{aligned}
+ & \frac{d \sqrt{6}}{5}\left(\frac{\rho\left(t_{2}\right)-(1-y) w_{s}^{2}-y w_{s+1}^{2}}{(1-y) q_{s}+y q_{s+1}} q(n)+w_{n}^{2}\right) \\
+ & \frac{d \sqrt{6}}{5}\left(\frac{\rho\left(t_{1}\right)-(1-y) w_{s}^{1}-y w_{s+1}^{1}}{(1-y) q_{s}+y q_{s+1}} q(n)+w_{n}^{1}\right) \\
& -\frac{2 d \sqrt{6}}{5}\left(\frac{\rho\left(t_{0}\right)-(1-y) w_{s}^{0}-y w_{s+1}^{0}}{(1-y) q_{s}+y q_{s+1}} q(n)+w_{n}^{0}\right) \\
& -\frac{d \tau \sqrt{6}}{10} q(n)+f\left(t_{2}-\frac{\tau}{2}, x_{n}\right), \quad 1 \leq n \leq M-1, \\
w_{0}^{2}= & w_{M}^{2}=0, \\
w_{n}^{0}= & \sin \left(x_{n}\right), \quad 0 \leq n \leq M .
\end{aligned}
$$

The system of linear equations given above can be written in the matrix form

$$
A_{2} w^{2}+B_{2} w^{1}+C_{2} w^{0}=D \varphi^{2}
$$

where

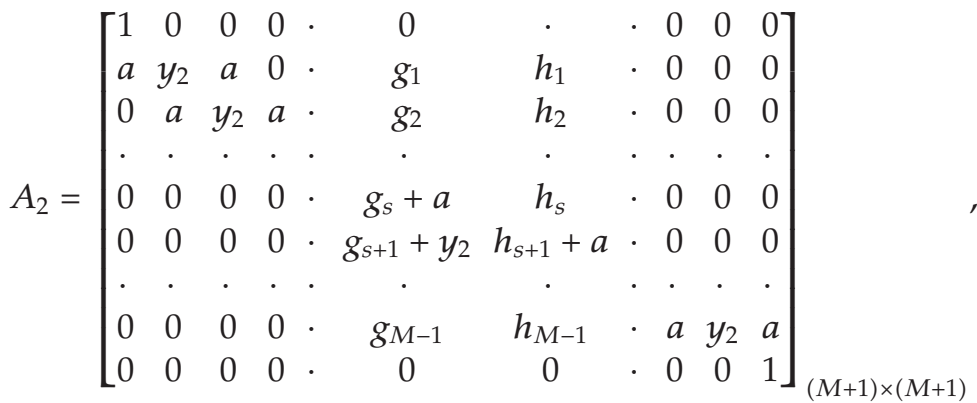

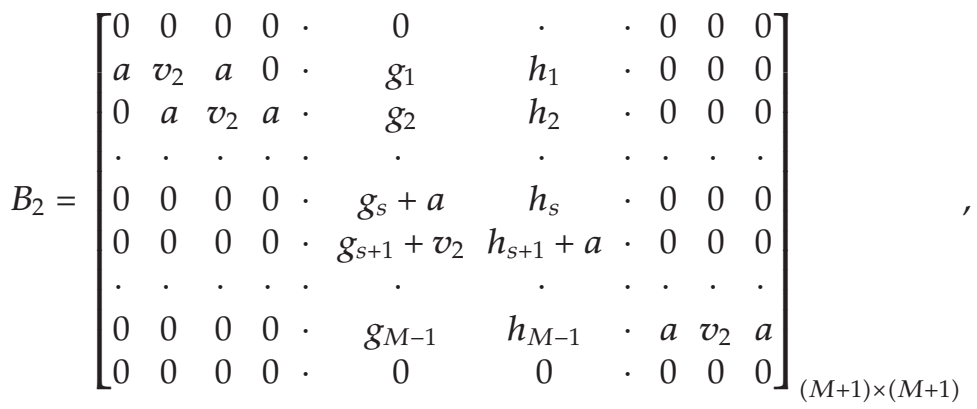


Abstract and Applied Analysis

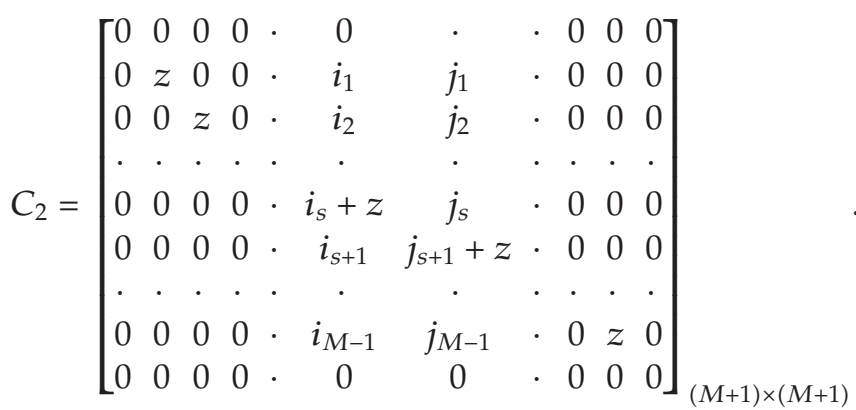

Here, for any $n=1,2, \ldots, M-1$,

$$
\begin{aligned}
& a=-\frac{1}{2 h^{2}}, \quad y_{2}=\frac{1}{\tau}+\frac{1}{h^{2}}+\frac{1}{2}-\frac{d \sqrt{6}}{5}, \\
& v_{2}=-\frac{1}{\tau}+\frac{1}{h^{2}}+\frac{1}{2}-\frac{d \sqrt{6}}{5} \\
& g_{n}=\frac{\left(q_{n+1}-2 q_{n}+q_{n-1}\right)(1-y)}{2 h^{2}\left((1-y) q_{s}+y q_{s+1}\right)}+\frac{q_{n}(1-y)}{2\left((1-y) q_{s}+y q_{s+1}\right)} \\
& +\frac{d \sqrt{6} q_{n}(1-y)}{5\left((1-y) q_{s}+y q_{s+1}\right)}, \quad \text { in }(s+1) \text { th column, } \\
& h_{n}=\frac{\left(q_{n+1}-2 q_{n}+q_{n-1}\right) y}{2 h^{2}\left((1-y) q_{s}+y q_{s+1}\right)}+\frac{q_{n} y}{2\left((1-y) q_{s}+y q_{s+1}\right)} \\
& +\frac{d \sqrt{6} q_{n} y}{5\left((1-y) q_{s}+y q_{s+1}\right)}, \quad \text { in }(s+2) \text { th column, } \\
& i_{n}=-\frac{2 d \sqrt{6} q_{n}(1-y)}{5\left((1-y) q_{s}+y q_{s+1}\right)}, \quad j_{n}=-\frac{2 \sqrt{6} q_{n} y}{5\left((1-y) q_{s}+y q_{s+1}\right)}, \quad z=\frac{2 d \sqrt{6}}{5}, \\
& \varphi^{2}=\left[\begin{array}{c}
0 \\
\phi_{1}^{2} \\
\vdots \\
\phi_{M-1}^{2} \\
0
\end{array}\right]_{(M+1) \times 1} \\
& \phi_{n}^{2}=\left(\frac{\sin \left(x_{n+1}\right)-2 \sin \left(x_{n}\right)+\sin \left(x_{n-1}\right)}{2 h^{2}}-\frac{\sin \left(x_{n}\right)}{2}+\frac{d \sqrt{6} q_{n}}{5}\right) \\
& \times \frac{\rho\left(t_{2}\right)+\rho\left(t_{1}\right)}{(1-y) q_{s}+y q_{s+1}}-\frac{2 d \sqrt{6} q_{n}}{5} \frac{\rho\left(t_{0}\right)}{(1-y) q_{s}+y q_{s+1}}-\frac{d \tau \sqrt{6}}{10} q_{n}+f\left(t_{2}-\frac{\tau}{2}, x_{n}\right) .
\end{aligned}
$$


Using (3.34), we can obtain that

$$
w^{2}=A_{2}^{-1}\left(D \varphi^{2}-B_{2} w^{1}-C_{2} w^{0}\right), \quad w^{0}=\left\{\sin x_{n}\right\}_{n=0}^{M} .
$$

For $3 \leq k \leq N$, we can obtain the following difference scheme:

$$
\begin{aligned}
& \frac{w_{n}^{k}-w_{n}^{k-1}}{\tau}=\frac{w_{n+1}^{k}-2 w_{n}^{k}+w_{n-1}^{k}}{2 h^{2}}+\frac{w_{n+1}^{k-1}-2 w_{n}^{k-1}+w_{n-1}^{k-1}}{2 h^{2}} \\
& -\frac{w_{n}^{k}+w_{n}^{k-1}}{2}+\left(\frac{q_{n+1}-2 q_{n}+q_{n-1}}{2 h^{2}}-\frac{q_{n}}{2}\right) \\
& \times\left(\frac{\rho\left(t_{k}\right)-(1-y) w_{s}^{k}-y w_{s+1}^{k}}{(1-y) q_{s}+y q_{s+1}}\right)+\left(\frac{q_{n+1}-2 q_{n}+q_{n-1}}{2 h^{2}}-\frac{q_{n}}{2}\right) \\
& \times\left(\frac{\rho\left(t_{k-1}\right)-(1-y) w_{s}^{k-1}-y w_{s+1}^{k-1}}{(1-y) q_{s}+y q_{s+1}}\right) \\
& +d \sum_{m=2}^{k-1}\left\{\left((k-m) b_{1}+b_{2}\right)\right. \\
& \times\left(\frac{\rho\left(t_{m-2}\right)-(1-y) w_{s}^{m-2}-y w_{s+1}^{m-2}}{(1-y) q_{s}+y q_{s+1}} q(n)\right)+\left((2 m-2 k-1) b_{1}-2 b_{2}\right) \\
& \times\left(\frac{\rho\left(t_{m-1}\right)-(1-y) w_{s}^{m-1}-y w_{s+1}^{m-1}}{(1-y) q_{s}+y q_{s+1}} q(n)\right) \\
& +\left((2 m-2 k-1) b_{1}-2 b_{2}\right) w_{n}^{m-1} \\
& \times\left(\frac{\rho\left(t_{m}\right)-(1-y) w_{s}^{m}-y w_{s+1}^{m}}{(1-y) q_{s}+y q_{s+1}} q(n)\right)+\left((k-m) b_{1}+b_{2}\right) w_{n}^{m-2} \\
& \left.+\left((k-m-1) b_{1}+b_{2}\right) w_{n}^{m}+\left((2 m-2 k-1) b_{1}-2 b_{2}\right) w_{n}^{m-1}\right\} \\
& -\frac{d}{6 \sqrt{2}}\left(\frac{\rho\left(t_{k-2}\right)-(1-y) w_{s}^{k-2}-y w_{s+1}^{k-2}}{(1-y) q_{s}+y q_{s+1}} q(n)+w_{n}^{k-2}\right) \\
& -\frac{4 d}{6 \sqrt{2}}\left(\frac{\rho\left(t_{k-1}\right)-(1-y) w_{s}^{k-1}-y w_{s+1}^{k-1}}{(1-y) q_{s}+y q_{s+1}} q(n)+w_{n}^{k-1}\right) \\
& +\frac{5 d}{6 \sqrt{2}}\left(\frac{\rho\left(t_{k}\right)-(1-y) w_{s}^{k}-y w_{s+1}^{k}}{(1-y) q_{s}+y q_{s+1}} q(n)+w_{n}^{k}\right)+f\left(t_{k}-\frac{\tau}{2}, x_{n}\right), \\
& 1 \leq n \leq M-1, \\
& w_{0}^{k}=w_{M}^{k}=0, \quad 3 \leq k \leq N, \\
& w_{n}^{0}=\sin \left(x_{n}\right), \quad 0 \leq n \leq M \text {. }
\end{aligned}
$$


This system can be written in matrix form as

$$
A_{3} w^{k}+B_{3} w^{k-1}+C_{3} w^{k-2}+\sum_{j=0}^{k-3} E_{j} w^{j}=D \varphi^{k}
$$

where

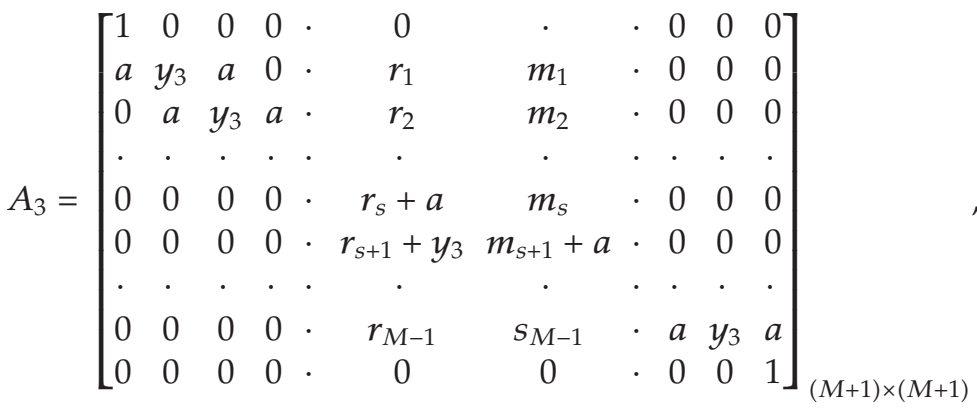

$$
\begin{aligned}
& B_{3}=\left[\begin{array}{ccccccccccc}
0 & 0 & 0 & 0 & \cdot & 0 & \cdot & \cdot & 0 & 0 & 0 \\
a & X & a & 0 & \cdot & Y_{1} & Z_{1} & \cdot & 0 & 0 & 0 \\
0 & a & X & a & \cdot & Y_{2} & Z_{2} & \cdot & 0 & 0 & 0 \\
\cdot & \cdot & \cdot & \cdot & \cdot & \cdot & \cdot & \cdot & \cdot & \cdot & \cdot \\
0 & 0 & 0 & 0 & \cdot & Y_{s}+a & Z_{s} & \cdot & 0 & 0 & 0 \\
0 & 0 & 0 & 0 & \cdot & Y_{s+1}+X & Z_{s+1}+a & \cdot & 0 & 0 & 0 \\
\cdot & \cdot & \cdot & \cdot & \cdot & \cdot & \cdot & \cdot & \cdot & \cdot & \cdot \\
0 & 0 & 0 & 0 & \cdot & Y_{M-1} & Z_{M-1} & \cdot & a & X & a \\
0 & 0 & 0 & 0 & \cdot & 0 & \cdot & \cdot & 0 & 0 & 0
\end{array}\right]_{(M+1) \times(M+1)}
\end{aligned}
$$

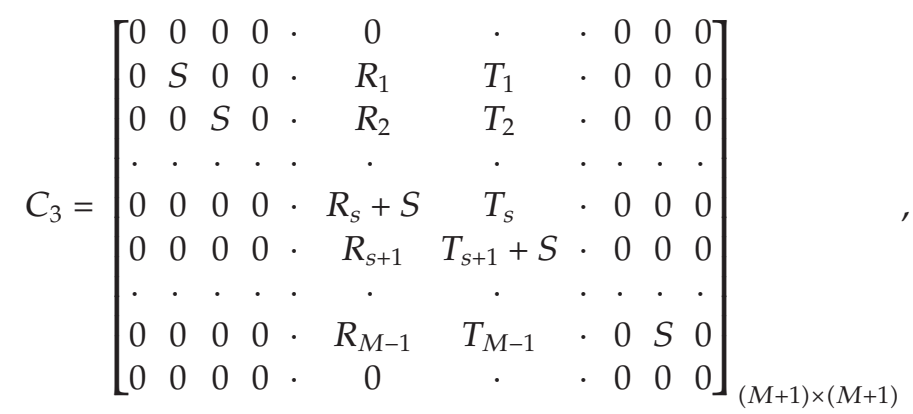

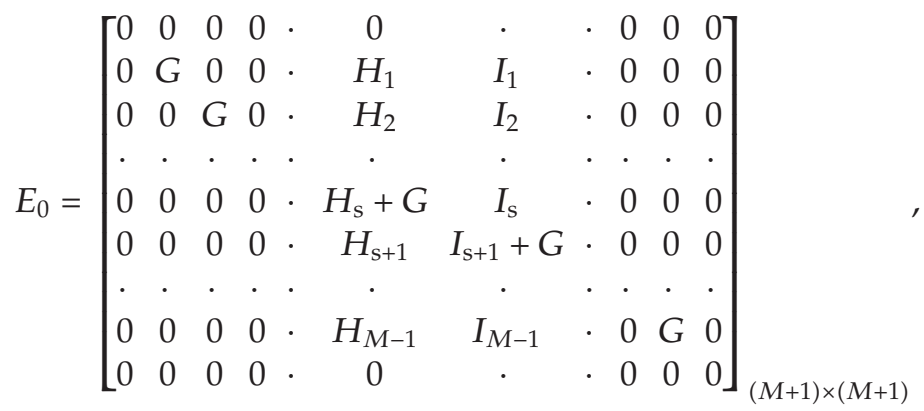




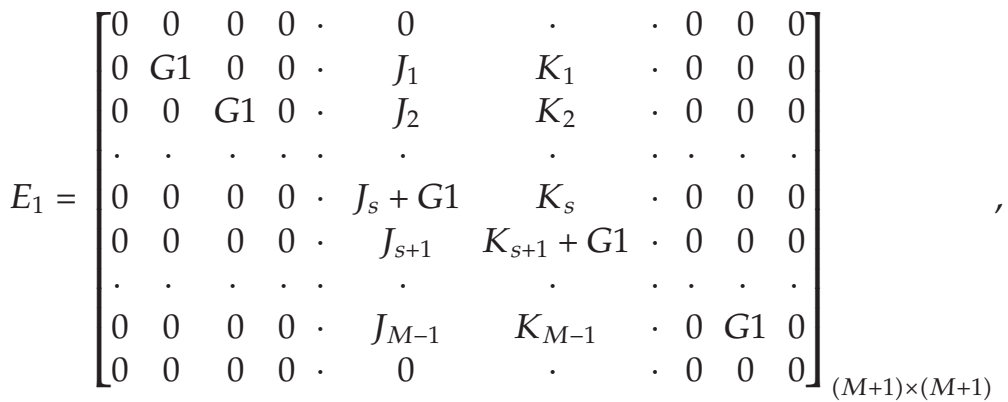

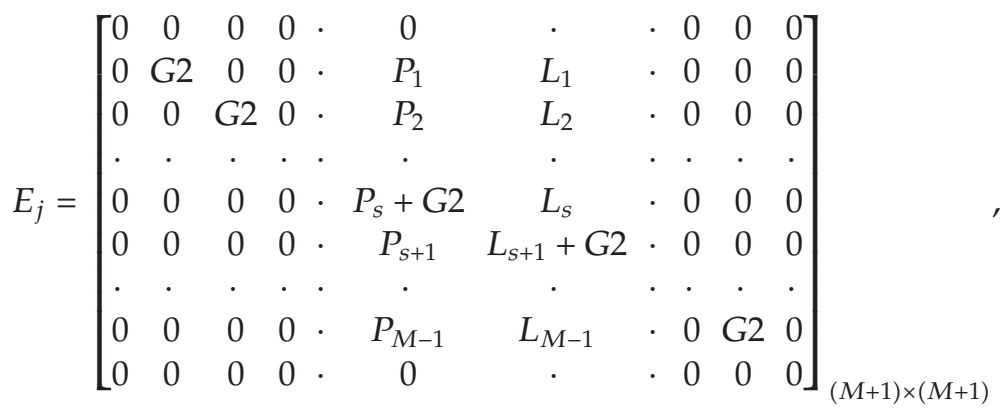

for $j=2,3, \ldots k-3$.

Here, for any $n=1,2, \ldots, M-1$,

$$
\begin{gathered}
a=-\frac{1}{2 h^{2}}, \quad d=\frac{2}{\sqrt{\pi \tau}}, \quad y_{3}=\frac{1}{\tau}+\frac{1}{h^{2}}+\frac{1}{2}-\frac{5 d}{6 \sqrt{2}}, \\
v_{3}=-\frac{1}{\tau}+\frac{1}{h^{2}}+\frac{1}{2}+\frac{4 d}{6 \sqrt{2}}, \\
\alpha_{n}=\frac{d q(n)(1-y)}{6 \sqrt{2}\left((1-y) q_{s}+y q_{s+1}\right)}, \quad \beta_{n}=\frac{d q(n) y}{6 \sqrt{2}\left((1-y) q_{s}+y q_{s+1}\right)}, \\
r_{n}=\frac{\left(q_{n+1}-2 q_{n}+q_{n-1}\right)(1-y)}{2 h^{2}\left((1-y) q_{s}+y q_{s+1}\right)}+\frac{q_{n}(1-y)}{2\left((1-y) q_{s}+y q_{s+1}\right)}+5 \frac{d q(n)(1-y)}{6 \sqrt{2}\left((1-y) q_{s}+y q_{s+1}\right)}, \\
s_{n}=\frac{\left(q_{n+1}-2 q_{n}+q_{n-1}\right) y}{2 h^{2}\left((1-y) q_{s}+y q_{s+1}\right)}+\frac{d q(n) y}{2\left((1-y) q_{s}+y q_{s+1}\right)}+5 \frac{q_{n} y}{6 \sqrt{2}\left((1-y) q_{s}+y q_{s+1}\right)}, \\
X=v_{3}-p p \times d, \quad Y_{n-1}=\gamma_{n-1}+6 \sqrt{2} p p \times \alpha_{n-1}, \\
Z_{n-1}=\delta_{n-1}+6 \sqrt{2} p p \times \beta_{n-1}, \quad S=d\left(\frac{1}{6 \sqrt{2}}-p p-n n\right), \\
R_{n-1}=-\alpha_{n-1}+6 \sqrt{2}(n n+p p) \alpha_{n-1}, \\
T_{n-1}=-\beta_{n-1}+6 \sqrt{2}(n n+p p) \beta_{n-1},
\end{gathered}
$$


Abstract and Applied Analysis

$$
\begin{aligned}
& \gamma_{n}=\frac{\left(q_{n+1}-2 q_{n}+q_{n-1}\right)(1-y)}{2 h^{2}\left((1-y) q_{s}+y q_{s+1}\right)}+\frac{q_{n}(1-y)}{2\left((1-y) q_{s}+y q_{s+1}\right)}-4 \frac{d q(n)(1-y)}{6 \sqrt{2}\left((1-y) q_{s}+y q_{s+1}\right)} \\
& \delta_{n}=\frac{\left(q_{n+1}-2 q_{n}+q_{n-1}\right) y}{2 h^{2}\left((1-y) q_{s}+y q_{s+1}\right)}+\frac{q_{n} y}{2\left((1-y) q_{s}+y q_{s+1}\right)}-4 \frac{d q(n) y}{6 \sqrt{2}\left((1-y) q_{s}+y q_{s+1}\right)} \\
& m m=(k-m) b_{1}+b_{2}, \quad n n=(2 m-2 k-1) b_{1}-2 b_{2}, \\
& p p=(k-m+1) b_{1}-2 b_{2} \\
& n n 1=(2(m+1)-2 k-1) b_{1}-2 b_{2} \\
& p p 1=(k-(m+2)+1) b_{1}+b_{2} \\
& G=-d \times m m, \quad G 1=-d \times(m m+n n 1), \\
& G 2=-d \times(m m+n n 1+p p 1), \\
& H_{n-1}=6 \sqrt{2} \times \alpha_{1} \times m m, \quad I_{n-1}=6 \sqrt{2} \times \beta_{1} \times m m, \\
& J_{n-1}=6 \sqrt{2} \times \alpha_{n-1} \times(m m+n n 1), \\
& K_{n-1}=6 \sqrt{2} \times \beta_{n-1} \times(m m+n n 1), \\
& P_{n-1}=6 \sqrt{2} \times \alpha_{n-1} \times(m m+n n 1+p p 1), \\
& L_{n-1}=6 \sqrt{2} \times \beta_{n-1} \times(m m+n n 1+p p 1), \\
& \varphi^{k}=\left[\begin{array}{c}
0 \\
\phi_{1}^{k} \\
\vdots \\
\phi_{M-1}^{k} \\
0
\end{array}\right]_{(M+1) \times 1}, \\
& \phi_{n}^{k}=\left(\frac{\sin \left(x_{n+1}\right)-2 \sin \left(x_{n}\right)+\sin \left(x_{n-1}\right)}{2 h^{2}}-\frac{\sin \left(x_{n}\right)}{2}\right) \frac{\rho\left(t_{k}\right)+\rho\left(t_{k-1}\right)}{(1-y) q_{s}+y q_{s+1}} \\
& +\frac{5 d q_{n} \rho\left(t_{k}\right)}{6 \sqrt{2}\left((1-y) q_{s}+y q_{s+1}\right)}-\frac{4 d q_{n} \rho\left(t_{k-1}\right)}{6 \sqrt{2}\left((1-y) q_{s}+y q_{s+1}\right)} \\
& -\frac{d q_{n} \rho\left(t_{k-2}\right)}{6 \sqrt{2}\left((1-y) q_{s}+y q_{s+1}\right)}+f\left(t_{k}-\frac{\tau}{2}, x_{n}\right)+\frac{d q_{n}}{(1-y) q_{s}+y q_{s+1}} \\
& \times \sum_{m=2}^{k-1}\left\{\left((k-m) b_{1}+b_{2}\right) \rho\left(t_{m-2}\right)\right. \\
& \left.+\left((2 m-2 k-1) b_{1}-2 b_{2}\right) \rho\left(t_{m-1}\right)+\left((k-m+1) b_{1}+b_{2}\right) \rho\left(t_{m}\right)\right\} .
\end{aligned}
$$


Table 1: Comparison of exact solution and approximate solutions.

\begin{tabular}{lccc}
\hline Method & $N=M=15$ & $N=M=45$ & $N=M=75$ \\
\hline 1st order of accuracy & 0.1190 & 0.0126 & 0.0045 \\
2nd order of accuracy & 0.0055 & $6.0917 \times 10^{-4}$ & $2.1932 \times 10^{-4}$ \\
\hline
\end{tabular}

Finally, from (3.39), it follows that

$$
w^{k}=\left(A_{3}\right)^{-1}\left(D \varphi^{k}-B_{3} w^{k-1}-C_{3} w^{k-2}-\sum_{j=0}^{k-3} E_{j} w^{j}\right), \quad 3 \leq k \leq N
$$

Applying the last formula step by step, we can reach $w^{k}$. Then, using (3.21), (3.25), and (3.26), we reach the approximate solutions of $u(t, x)$ and $\left(p\left(t_{k}\right)+p\left(t_{k-1}\right)\right) / 2$.

\subsection{Error Analysis}

In this part, the results of the numerical analysis is given. The numerical solutions are recorded for different values of $N$ and $M$ and $u_{n}^{k}$ represents the approximate solution of $u(t, x)$ at grid points $\left(t_{k}, x_{n}\right)$. Table 1 gives the error analysis between the exact solution and the solutions derived by difference schemes. Table 1 is constructed for $N=M=15,45$, and 75 , respectively. For their comparison, the errors are computed by

$$
E=\max _{1 \leq k \leq N_{1 \leq n \leq M}}\left|u\left(t_{k}, x_{n}\right)-u_{n}^{k}\right|
$$

Thus, the second order of accuracy difference scheme is more accurate comparing with the first order of accuracy difference scheme.

\section{Acknowledgment}

The authors are grateful to Professor Allaberen Ashyralyev (Fatih University, Turkey) for his comments and suggestions to improve the quality of the paper.

\section{References}

[1] A. Hasanov, "Identification of unknown diffusion and convection coefficients in ion transport problems from flux data: an analytical approach," Journal of Mathematical Chemistry, vol. 48, no. 2, pp. 413-423, 2010.

[2] A. Hasanov and S. Tatar, "An inversion method for identification of elastoplastic properties of a beam from torsional experiment," International Journal of Non-Linear Mechanics, vol. 45, pp. 562-571, 2010.

[3] G. Di Blasio and A. Lorenzi, "Identification problems for parabolic delay differential equations with measurement on the boundary," Journal of Inverse and Ill-Posed Problems, vol. 15, no. 7, pp. 709-734, 2007.

[4] D. Orlovsky and S. Piskarev, "On approximation of inverse problems for abstract elliptic problems," Journal of Inverse and Ill-Posed Problems, vol. 17, no. 8, pp. 765-782, 2009.

[5] Y. S. Eidelman, "A boundary value problem for a differential equation with a parameter," Differentsia'nye Uravneniya, vol. 14, no. 7, pp. 1335-1337, 1978. 
[6] A. Ashyralyev, "On a problem of determining the parameter of a parabolic equation," Ukranian Mathematical Journal, vol. 62, no. 9, pp. 1200-1210, 2010.

[7] V. Serov and L. Päivärinta, "Inverse scattering problem for two-dimensional Schrödinger operator," Journal of Inverse and Ill-Posed Problems, vol. 14, no. 3, pp. 295-305, 2006.

[8] V. T. Borukhov and P. N. Vabishchevich, "Numerical solution of the inverse problem of reconstructing a distributed right-hand side of a parabolic equation," Computer Physics Communications, vol. 126, no. 1-2, pp. 32-36, 2000.

[9] A. A. Samarskii and P. N. Vabishchevich, Numerical Methods for Solving Inverse Problems of Mathematical Physics, Inverse and Ill-Posed Problems Series, Walter de Gruyter, Berlin, Germany, 2007.

[10] A. I. Prilepko and A. B. Kostin, "Some inverse problems for parabolic equations with final and integral observation," Matematicheskiu Sbornik, vol. 183, no. 4, pp. 49-68, 1992.

[11] I. Podlubny, Fractional Differential Equations, vol. 198 of Mathematics in Science and Engineering, Academic Press, San Diego, Calif, USA, 1999.

[12] S. G. Samko, A. A. Kilbas, and O. I. Marichev, Fractional integrals and derivatives, Gordon and Breach Science Publishers, Yverdon, Switzerland, 1993.

[13] A. A. Kilbas, H. M. Sristava, and J. J. Trujillo, Theory and Applications of Fractional Differential Equations, North-Holland Mathematics Studies, Elsevier Science, 2006.

[14] J.-L. Lavoie, T. J. Osler, and R. Tremblay, "Fractional derivatives and special functions," SIAM Review, vol. 18, no. 2, pp. 240-268, 1976.

[15] V. E. Tarasov, "Fractional derivative as fractional power of derivative," International Journal of Mathematics, vol. 18, no. 3, pp. 281-299, 2007.

[16] R. Gorenflo and F. Mainardi, "Fractional calculus: integral and differential equations of fractional order," in Fractals and Fractional Calculus in Continuum Mechanics, A. Carpinteri and F. Mainardi, Eds., vol. 378 of CISM Courses and Lectures, pp. 223-276, Springer, Vienna, Austria, 1997.

[17] D. Matignon, "Stability results for fractional differential equations with applications to control processing," in Computational Engineering in System Application, vol. 2, Lille, France, 1996.

[18] A. B. Basset, "On the descent of a sphere in a viscous liquid," Quarterly Journal of Mathematics, vol. 42, pp. 369-381, 1910.

[19] F. Mainardi, "Fractional calculus: some basic problems in continuum and statistical mechanics," in Fractals and Fractional Calculus in Continuum Mechanics, A. Carpinteri and F. Mainardi, Eds., vol. 378 of CISM Courses and Lectures, pp. 291-348, Springer, New York, NY, USA, 1997.

[20] A. Ashyralyev, F. Dal, and Z. Pinar, "On the numerical solution of fractional hyperbolic partial differential equations," Mathematical Problems in Engineering, vol. 2009, Article ID 730465, 11 pages, 2009.

[21] A. Ashyralyev, "A note on fractional derivatives and fractional powers of operators," Journal of Mathematical Analysis and Applications, vol. 357, no. 1, pp. 232-236, 2009.

[22] A. Ashyralyev and B. Hicdurmaz, "A note on the fractional Schrödinger differential equations," Kybernetes, vol. 40, no. 5-6, pp. 736-750, 2011.

[23] Y. Zhang, "A finite difference method for fractional partial differential equation," Applied Mathematics and Computation, vol. 215, no. 2, pp. 524-529, 2009.

[24] E. Cuesta, C. Lubich, and C. Palencia, "Convolution quadrature time discretization of fractional diffusion-wave equations," Mathematics of Computation, vol. 75, no. 254, pp. 673-696, 2006.

[25] P. E. Sobolevskii, "Some properties of the solutions of differential equations in fractional spaces," Trudy Naucno-Issledovatel'skogi Instituta Matematiki VGU, vol. 14, pp. 68-74, 1975 (Russian).

[26] G. Da Prato and P. Grisvard, "Sommes d'opérateurs linéaires et équations différentielles opérationnelles," Journal de Mathématiques Pures et Appliquées, vol. 54, no. 3, pp. 305-387, 1975.

[27] A. Ashyralyev and Z. Cakir, "On the numerical solution of fractional parabolic partial differential equations with the Dirichlet condition," in Proceedings of the 2 nd International Symposium on Computing in Science and Engineering (ISCSE '11), M. Gunes, Ed., pp. 529-530, Kusadasi, Aydın, Turkey, June 2011.

[28] A. Ashyralyev, "Well-posedness of the Basset problem in spaces of smooth functions," Applied Mathematics Letters, vol. 24, no. 7, pp. 1176-1180, 2011.

[29] D. A. Murio and C. E. Mejía, "Generalized time fractional IHCP with Caputo fractional derivatives," in Proceedings of the 6th International Conference on Inverse Problems in Engineering: Theory and Practice, vol. 135 of Journal of Physics: Conference Series, pp. 1-8, Dourdan, Paris, France, 2008.

[30] J. Cheng, J. Nakagawa, M. Yamamoto, and T. Yamazaki, “Uniqueness in an inverse problem for a one-dimensional fractional diffusion equation," Inverse Problems, vol. 25, no. 11, pp. 1-16, 2009. 
[31] J. Nakagawa, K. Sakamoto, and M. Yamamoto, "Overview to mathematical analysis for fractional diffusion equations-new mathematical aspects motivated by industrial collaboration," Journal of Math-for-Industry, vol. 2A, pp. 99-108, 2010.

[32] Y. Zhang and X. Xu, "Inverse source problem for a fractional diffusion equation," Inverse Problems, vol. 27, no. 3, pp. 1-12, 2011.

[33] K. Sakamoto and M. Yamamoto, "Inverse source problem with a final overdetermination for a fractional diffusion equation," Mathematical Control and Related Fields, vol. 1, no. 4, pp. 509-518, 2011.

[34] A. Ashyralyev, "A note on fractional derivatives and fractional powers of operators," Journal of Mathematical Analysis and Applications, vol. 357, no. 1, pp. 232-236, 2009.

[35] A. Ashyralyev and P. E. Sobolevskii, New Difference Schemes for Partial Differential Equations, vol. 148 of Operator Theory: Advances and Applications, Birkhäuser Verlag, Berlin, Germany, 2004.

[36] A. Ashyralyev, "Fractional spaces generated by the positive differential and difference operators in a Banach space," in Mathematical Methods in Engineering, K. Taş, J. A. Tenreiro Machado, and D. Baleanu, Eds., pp. 13-22, Springer, Dordrecht, The Netherlands, 2007. 


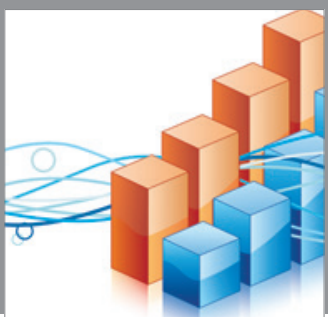

Advances in

Operations Research

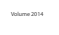

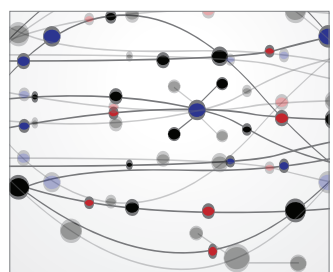

\section{The Scientific} World Journal
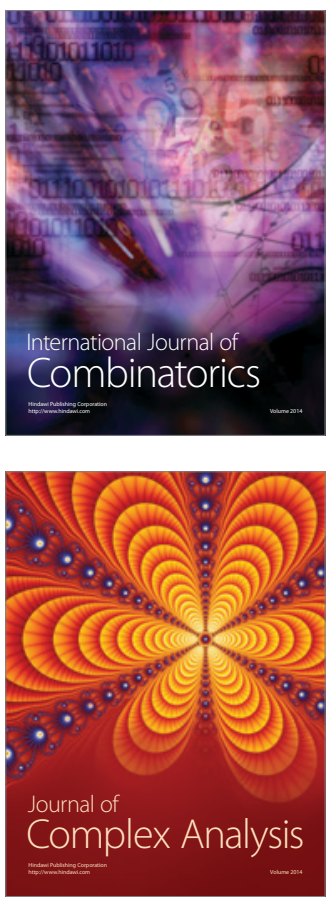

International Journal of

Mathematics and

Mathematical

Sciences
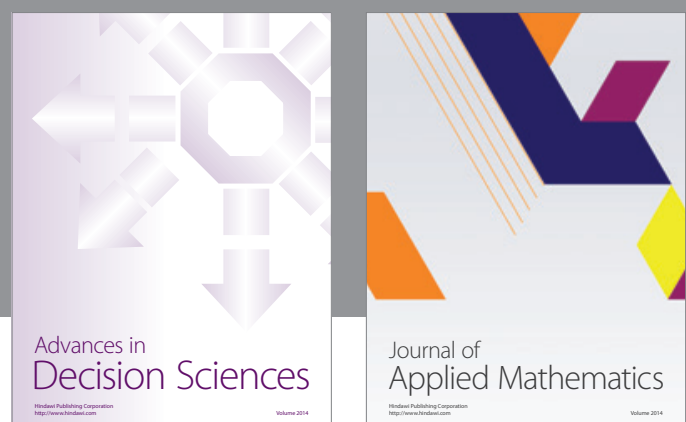

Journal of

Applied Mathematics
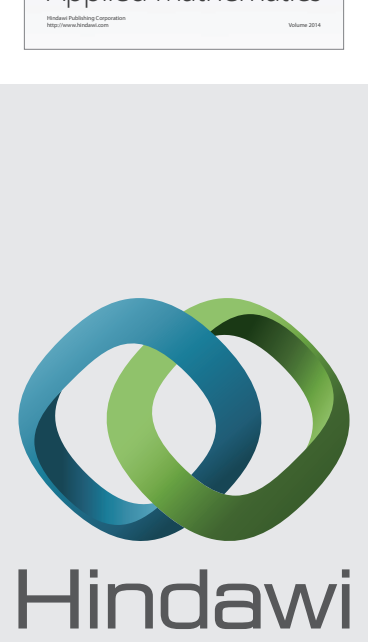

Submit your manuscripts at http://www.hindawi.com
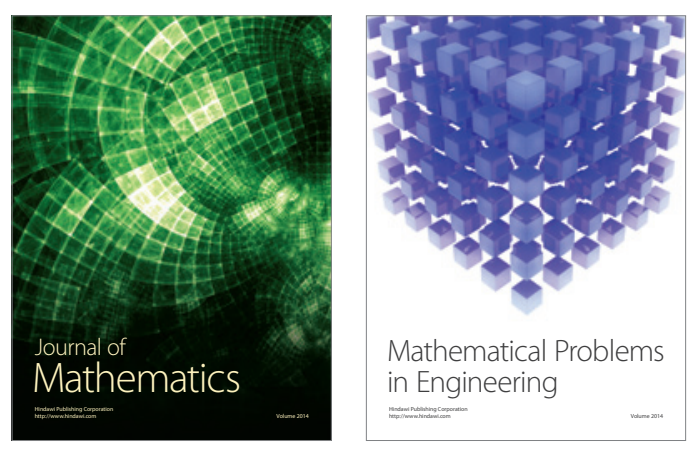

Mathematical Problems in Engineering
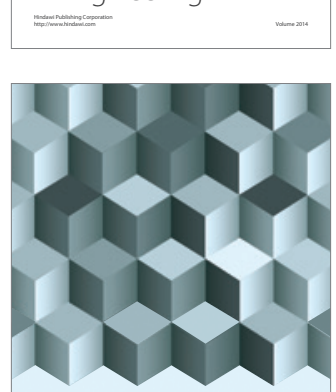

Journal of

Function Spaces
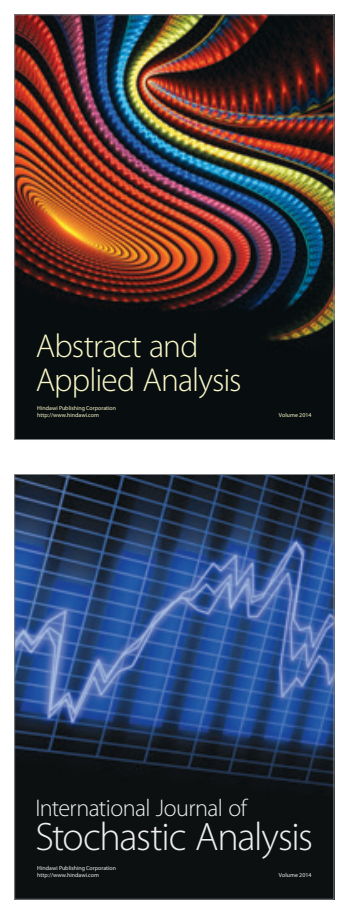

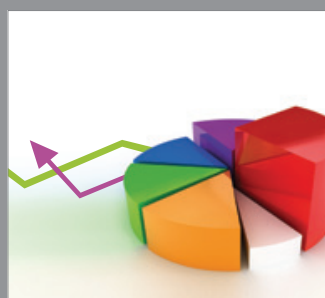

ournal of

Probability and Statistics

Promensencen
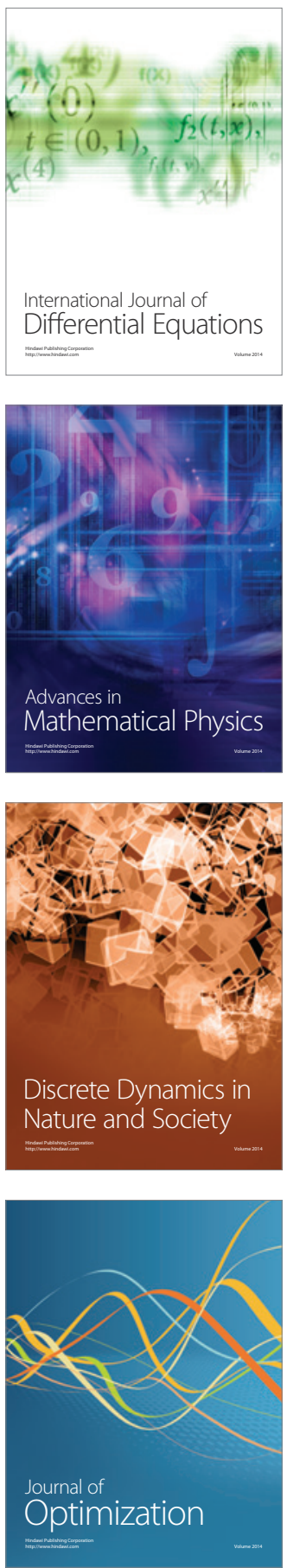